\title{
Considerations on the Graviton Excitation Modes of Hořava-Lifshitz Gravity
}

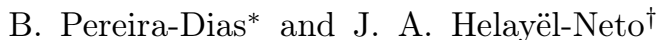 \\ Centro Brasileiro de Pesquisas Físicas (CBPF), \\ Rua Dr. Xavier Sigaud 150, Urca, \\ Rio de Janeiro, RJ, Brazil, CEP 22.290-180 \\ C. A. Hernask 丹 $^{\text {}}$ \\ Universidade Federal do Amapá (Unifap), \\ Rod. Juscelino Kubitschek KM-02, Jardim Marco Zero, \\ Macapá, AP, Brazil, CEP 68.902-280
}

\begin{abstract}
A new set of projection operators is constructed to suitably handle non-relativistic theories of gravity with anisotropic scaling, including the ones with parity-violating terms. This alternative procedure allows us to discuss unitarity and spectral properties for different formulations of the Hořava-Lifshitz gravity. This task, that generally involves lengthy algebraic steps, becomes more systematic and greatly simplified in terms of the projectors we work out. Moreover, this procedure allows us to fix the number of propagating degrees of freedom and the structure of gauge symmetries is readily determined. In order to test the efficacy of the technique at hand, the unitarity and lowenergy regime of a general Hořava-Lifshitz Gravity model are investigated.
\end{abstract}

PACS numbers: 04.50.Kd, 04.60.-m, 11.25.Db

\section{INTRODUCTION}

The recent proposal of Petr Hořava for a new theory of gravity [1-4] was received with great enthusiasm by the Field Theory, Gravitation and Cosmology communities [5-39] and interesting consequences for cosmological models have been worked out [40 47]. The large appraisal for this model, dubbed as Hořava-Lifshitz gravity (HLG), came from the fact that it is a candidate for a consistent quantum field theory for gravity, since it has a remarkably improved ultraviolet behavior. However, to achieve such a result, Hořava had to give up diffeomorphism invariance as a fundamental symmetry so to impose anisotropic scaling of the space and time dimensions.

Despite its success, it soon became evident that Hořava's original formulation including the detailed balance condition is not obviously a phenomenologically viable model [15, 31, 32. So, to reconcile HLG with experimentation, it became necessary to loose up such condition and allow the inclusion of a large number of Lagrangian terms with arbitrary coefficients in the model or introduce auxiliary fields to enlarge the symmetries of the model. Besides this, HLG can be formulated in two distinct versions: nonprojectable or projectable - which refers to the dependence or not of the lapse function with the space coordinates. These different possible formulations brought a discussion in the literature concerning whether each of these formulations should be considered as a good, bad, healthy, or ugly version of HLG [9, 27].

All this debate included not only analysis of the phenomenological aspect, but also considerations on the the quantum consistency of each version of theory. It is in this context that we judge necessary (and convenient) to develop a general method for the attainment of the propagators and, thereby, the identification of the excitations present in the possible models based on the HLG frame. With the propagators at hand it is possible to systematically obtain a description of the particle spectrum and the relativistic and quantum properties of scattering processes. More exactly, one is able to pinch out the propagating modes and determine conditions on the coefficients of the Lagrangian so as to impose the absence of tachyons and ghosts.

There are several methods for the attainment of propagators, but, particularly in the case of weak field approximation for quantum gravity, which is our interest, algebraic methods have been intensively developed, specially the one based on the spin projection operators (SPO) [48 50]. The SPO for Lorentz invariant models has the interesting property of being a orthonormal basis that decomposes the fields into definite spin-parity sectors. In this paper we follow the lines of previous works [51 54] to build up an orthonormal basis of operators that is specially suitable to handle of

\footnotetext{
*Electronic address: bpdias@cbpf.br

$\dagger$ Electronic address: helayel@cbpf.br

${ }^{\ddagger}$ Electronic address: carlos@cbpf.br
} 
HLG models, including the ones with parity-breaking terms. With this result we may discuss the spectral properties of the HLG and understand better the extra 3D spin-0 propagating mode that appear in the theory. Moreover, this technology allows us to determine the number of propagating degrees of freedom and the gauge symmetry structure is easily determined. We would like to point out that recently F. S. Bemfica and M. Gomes also discuss the propagators and consistency conditions on the spectrum for special classes of HLG models by adopting an independent method [5, 6].

This paper is organized as follows: In Sec. II we build up the basis of projection operators suitable with the symmetries of HLG. We present in the Sec. III a general method for the consistency analysis of the particle spectrum. The Lagrangian model of our interest shall be described in the Sec. [V] and the attainment of its propagators along with the spectral analysis is done is Sec. V. We present our Concluding Assessment in Sec. VI emphasizing in some consequences of the propagator structure of HLG in the low energy regime. In the Appendix A, we list the inverse of the spin-0 matrix used to calculate the propagators of Sec. V], whereas the Appendix B is aimed to derive some useful algebraic relations that the projection operators satisfy.

Unless stated otherwise, we shall use Latin letters $(a, b, c, \ldots)$ for $3 \mathrm{D}$ spatial index. We also define $\delta_{a b}=\operatorname{diag}(1,1,1)$ and $\epsilon_{a b c}$ as the completely antisymmetric symbol normalized to $\epsilon_{012}=+1$. Also, we shall follow the conventions: $R_{b c d}^{a}=\partial_{c} \Gamma_{b d}^{a}+\Gamma_{c e}^{a} \Gamma_{b d}^{e}-(c \leftrightarrow d), \Gamma_{b c}^{a}=\frac{1}{2} g^{a d}\left(\partial_{b} g_{d c}+\partial_{c} g_{d b}-\partial_{d} g_{b c}\right), R_{b d}=R_{b a d}^{a}, R=g^{b d} R_{b d}$, with $g_{a b}$ being the spatial metric.

\section{DECOMPOSING THE GRAVITON EXCITATION MODES}

In the Hořava-Lifshitz gravity (HLG), the breaking of $(1+3)$-dimensional diffeomorphisms invariance is implemented by endowing space-time with a preferred foliation of three-dimensional spacelike surfaces. This defines the splitting of coordinates into space and time that explicitly breaks general covariance down to the subgroup of coordinate transformations

$$
t \mapsto \tilde{t}(t), \quad x^{a} \mapsto \tilde{x}^{a}\left(t, x^{a}\right)
$$

referred in the literature as foliation-preserving diffeomorphisms.

To fulfill the task of defining a Lagrangian model with such a symmetry, it is natural to make use of the ADM (Arnowitt-Deser-Misner) decomposition, which in the convention we are adopting, decomposes the space-time metric ${ }^{(4)} g_{\mu \nu}$ in terms of the lapse $\mathcal{N}$, the shift $N_{a}$, and the spatial metric $g_{a b}$ as

$$
d s^{2}=\left(\mathcal{N}^{2}-N_{a} N^{a}\right) d t^{2}-2 N_{a} d x^{a} d t-g_{a b} d x^{a} d x^{b}
$$

From the equation (2) we may relate the inverse of the four-dimensional metric and the ADM fields by

$$
{ }^{(4)} g^{00}=\frac{1}{\mathcal{N}^{2}}, \quad{ }^{(4)} g^{0 a}=-\frac{N^{a}}{\mathcal{N}^{2}}, \quad{ }^{(4)} g^{a b}=-g^{a b}+\frac{N^{a} N^{b}}{\mathcal{N}^{2}},
$$

and $\sqrt{-\operatorname{det}^{(4)} g_{\mu \nu}}=\mathcal{N} \sqrt{\operatorname{det} g_{a b}}$.

Having defined the fundamental fields of the model, we may perform a weak field expansion around a Minkowski metric solution in order to study the graviton excitation modes of HLG,

$$
\mathcal{N}=1+n, \quad g_{a b}=\delta_{a b}+h_{a b},
$$

with $n$ and $h_{a b}$ being the quantum fluctuation of the lapse function and the spatial metric, respectively, and the shift function $N_{a}$ being the quantum fluctuation itself.

Breaking down the Minkowski symmetry, $S O(1,3)$, of the linearized theory to a $S O(3)$ has some nontrivial consequences, specially in the interpretation of the propagating modes. An usual relativistic quantum field theory is invariant under Poincaré transformations. It provides a classification for particles as unitary irreducible representations of the Poincaré group by the quantum numbers: mass and spin. In particular, the spin is characterized by the unitary representations of the $S O(1,3)$ little group, that is the subgroup of the Lorentz group that leaves the representative four-momentum unchanged.

For the class of HLG models, we may proceed in a strict analogy. We split our propagating modes in "what would be the spin" defined the subgroup of the rotation group which leaves the representative three-momentum invariant. So, to arrange the degree-of-freedom into these "spin modes", we may develop a complete set of projection operators for Hořava-like models. For this task, it is reasonable to make use of the already developed three-dimensional $S O(1,2)$ 
Barnes-Rivers projection operators [51] and adapt them for a $S O(3)$-invariant model implementing the following substitution:

$$
\eta_{\hat{\mu} \hat{\nu}} \rightarrow \delta_{a b}, \quad k_{\hat{\mu}} \rightarrow k_{a}
$$

where $\hat{\mu}, \hat{\nu}=0,1,2, a, b=1,2,3, k_{\hat{\mu}}$ is the $(1+2)-\mathrm{D}$ relativistic momentum and $k_{a}$ is the $3 \mathrm{D}$ spatial momentum. For example, in the given situation, the transverse $\left(\theta_{a b}\right)$ and the longitudinal $\left(\omega_{a b}\right)$ operators, which are building blocks operators become

$$
\begin{aligned}
\theta_{a b} & =\delta_{a b}-\omega_{a b}, \\
\omega_{a b} & =\frac{k_{a} k_{b}}{k^{2}}
\end{aligned}
$$

with $k^{2}=k_{a} k_{a}$.

In this way, we may organize the HLG projection operators in a matrix-form according to the respective spin sector $(P(0), P(1)$, and $P(2))$ to cover the case of parity-preserving Lagrangians containing the fields $n, N_{a}$, and $h_{a b}$. They are arranged by the possible spin representations carried in the quantum fields $-n \in \underline{0}, N_{a} \in \underline{0} \oplus \underline{1}, h_{a b} \in \underline{0} \oplus \underline{1} \oplus \underline{2}$, which comes from usual group-theoretic argumentation. So, we cast them as

$$
\begin{aligned}
& P(0)=\begin{array}{c}
h_{a b} \\
h_{a b} \\
N_{a} \\
n
\end{array}\left[\begin{array}{cccc}
h_{c d} & h_{c d} & N_{c} & { }^{n} \\
\frac{1}{2} \theta_{a b} \theta_{c d} & \frac{1}{\sqrt{2}} \theta_{a b} \omega_{c d} & \frac{1}{\sqrt{2}} \theta_{a b} \bar{k}_{c} & \frac{1}{\sqrt{2}} \theta_{a b} \\
\frac{1}{\sqrt{2}} \omega_{a b} \theta_{c d} & \omega_{a b} \omega_{c d} & \omega_{a b} \bar{k}_{c} & \omega_{a b} \\
\frac{1}{\sqrt{2}} \bar{k}_{a} \theta_{b c} & \bar{k}_{a} \omega_{b c} & \omega_{a b} & \bar{k}_{a} \\
\frac{1}{\sqrt{2}} \theta_{c d} & \omega_{c d} & \bar{k}_{b} & 1
\end{array}\right] \\
& P(1)=h_{a b}\left[\begin{array}{cc}
h_{c d} & N_{a} \\
N_{a}
\end{array}\left[\begin{array}{cc}
\left.N_{a c} \omega_{b d}+\theta_{b c} \omega_{a d}+\theta_{a d} \omega_{b c}+\theta_{b d} \omega_{a c}\right) & \frac{1}{\sqrt{2}}\left(\theta_{a c} \bar{k}_{b}+\theta_{b c} \bar{k}_{a}\right) \\
\frac{1}{\sqrt{2}}\left(\theta_{b a} \bar{k}_{c}+\theta_{c a} \bar{k}_{b}\right) & \theta_{a b}
\end{array}\right]\right. \\
& P^{h h}(2)=\frac{1}{2}\left(\theta_{a c} \theta_{b d}+\theta_{a d} \theta_{b c}-\theta_{a b} \theta_{c d}\right)
\end{aligned}
$$

where we have defined $\bar{k}_{a}=\frac{k_{a}}{\sqrt{k^{2}}}$.

However, even in Hořava's original proposal with detailed balance and its subsequent modifications there appear parity violating terms, that notably cannot be written in terms of the $S O(3)$ Barnes-Rivers operators. A hint to circumvent this difficulty is to note that in the presence of parity violating terms, the transverse modes may propagate independently and not as parity-doublets (as discussed in Ref. [52] and put into test in Ref. [53]). In practice, the two degrees of freedom of the $\theta$-operator can be split by two orthonormal operators,

$$
\theta_{a b}=\rho_{a b}+\sigma_{a b}
$$

satisfying $\rho^{2}=\rho, \sigma^{2}=\sigma$, and $\rho \cdot \sigma=0$. This decomposition is useful to handle with parity violating term such as the ones containing the $\epsilon$-symbol, since $\rho$ and $\sigma$ may be defined to satisfy

$$
\epsilon^{a b c} k_{c}=i \sqrt{k^{2}}\left(\rho_{a b}-\sigma_{a b}\right)
$$

The degree-of-freedom projection operators for relativistic planar models $(S O(1,2)$-invariant theories $)$ with paritybreaking terms have been thoroughly worked out in the Ref. [52] and can be used adequately for HLG models with parity violating terms. Specializing these projection operators for the fields of the ADM decomposition, one obtains,

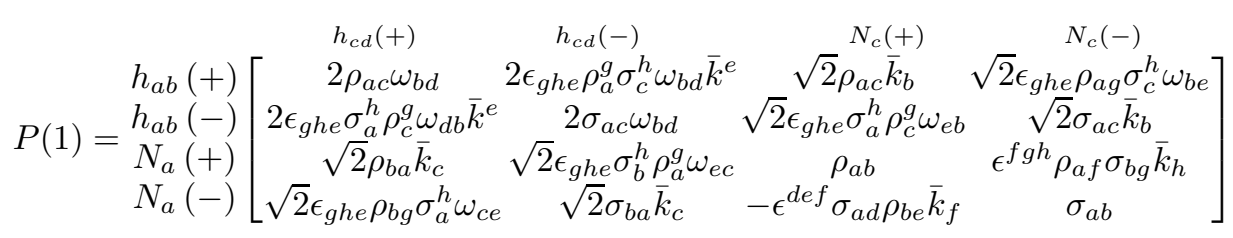




$$
P(2)=\begin{gathered}
h_{a b}(+) \\
h_{a b}(-)
\end{gathered}\left[\begin{array}{cc}
\frac{1}{2}\left(\rho_{a d} \rho_{b c}+\sigma_{a d} \sigma_{b c}-\rho_{a b} \sigma_{c d}-\sigma_{a b} \rho_{c d}\right) & \epsilon_{g h e}\left(\rho_{c a} \sigma_{d}^{h} \rho_{b}^{g}-\sigma_{c b} \rho_{d}^{g} \sigma_{a}^{h}\right) \bar{k}^{e} \\
\epsilon_{g h e}\left(\rho_{a c} \sigma_{b}^{h} \rho_{d}^{g}-\sigma_{a d} \rho_{b}^{g} \sigma_{c}^{h}\right) \bar{k}^{e} & 2 \rho_{a c} \sigma_{b d}
\end{array}\right]
$$

In the above matrices it is understood the operators share the same symmetrization properties (with numerical factor) of the associated fields.

It is noteworthy to emphasize that all the HLG projection operators (7a $)-(7 \mathrm{c})$ and (10a)-(10b) have been constructed in such a way that the orthogonality relationship and decomposition of unity relations are fulfilled:

$$
\begin{aligned}
\sum_{\beta} P_{i j}(I)_{\alpha \beta} P_{k l}(J)_{\beta \gamma} & =\delta_{j k} P_{i l}(I)_{\alpha \gamma}, \quad I, J=0,1,2 \\
\sum_{i, J} P_{i i}(J)_{\alpha \beta} & =\delta_{\alpha \beta},
\end{aligned}
$$

where the Greek indices $\alpha, \beta, \gamma, \ldots$ represents here either tensorial, vector or no spatial indices.

\section{ANALYSING THE CONSISTENCY OF THE PARTICLE SPECTRUM FOR A GENERAL MODEL}

To understand the convenience of orthonormal basis of operators for the attainment of the propagators, let us work out a general spectral analysis for models suitable with the symmetries defined by HLG models. For such, let $\mathcal{L}_{2}$ be a quadratic Lagrangian for a HLG model:

$$
\mathcal{L}_{2}=\sum_{\alpha \beta} \varphi_{\alpha} \mathcal{O}_{\alpha \beta} \varphi_{\beta}+\sum_{\alpha} \varphi_{\alpha} \mathcal{S}_{\alpha}
$$

where the wave operator $\mathcal{O}_{\alpha \beta}$ is a local differential operator, $\varphi_{\alpha}=\left\{n, N_{a}, h_{a b}\right\}$ carry the fundamental quantum fields of the model, and $\mathcal{S}_{\alpha}=\left\{\mathcal{S}, \mathcal{S}_{a}, \mathcal{S}_{a b}\right\}$ are the matter sources of the lapse, shift, and spatial metric, respectively. We can systematically analyze the spectrum and unitarity of this model by means of a decomposition of the wave operator $\mathcal{O}_{\alpha \beta}$ in terms of the HLG projection operators $P_{i j}^{\varphi \psi}(J)$ in the momentum space:

$$
\mathcal{L}_{2}=\sum_{\alpha \beta, i j, J} \varphi_{\alpha} a(J)_{i j} P_{i j}^{\varphi \psi}(J)_{\alpha \beta} \psi_{\beta}
$$

where $a(J)_{i j}$ are the coefficients of the expansion of the wave operator. The diagonal operators $P_{i i}^{\varphi \varphi}(J)$ are projectors in the spin- $J$ sector of the field $\varphi$ and the off-diagonal operators $P_{i j}^{\varphi \psi}(J)(i \neq j)$ implement mappings inside the spin subspace among the fields $\varphi$ and $\psi$.

The properties 11a - 11b of the HLG projection operators reduces the task of inverting the wave operator into a straightforward algebraic exercise. Indeed, if the coefficient matrices $a(J)_{i j}$ are invertible, then the propagator saturated with the external sources $\mathcal{S}_{\alpha}$ is given by

$$
\Pi=i \sum_{\alpha \beta, i j, J} \mathcal{S}_{\alpha}^{*} a(J)_{i j}^{-1} P_{i j}(J)_{\alpha \beta} \mathcal{S}_{\beta}^{\prime}
$$

Nevertheless, the coefficient matrices $a(J)_{i j}$ may become degenerate due to a gauge symmetry of the Lagrangian model. This poses no serious problem to the attainment of the propagator since, in a such case, there are sources constraints that consistently appear in order to inhibit the propagation of non-physical modes. Both, the gauge transformations of the fields and the source constraints may be obtained from the degeneracy structures of the coefficient matrices,

$$
\begin{aligned}
& \delta \phi_{\alpha}=\sum_{\beta, J, i} V_{i}^{(R, n)}(J) P_{i j}(J)_{\alpha \beta} f_{\beta}(J), \text { for any } j \\
& \sum_{\beta, j} V_{j}^{(L, n)}(J) P_{i j}(J)_{\alpha \beta} \mathcal{S}_{\beta}^{\prime}(J)=0, \text { for every } i \text { and } J
\end{aligned}
$$


with $f_{\beta}(J)$ being arbitrary functions and $V^{(R, n)}\left(V^{(L, n)}\right)$ being the right (left) null eigenvectors of $a(J)_{i j}$.

With it in mind, one should be convinced that the correct procedure for the attainment of the propagator is to invert any largest nondegenerate submatrix of $a(J)_{i j}$. In practice, it suffices to delete the degenerate rows and columns of $a(J)_{i j}$ (which we denote $A(J)_{i j}$ ) according to the number of gauge symmetries, to invert $A(J)_{i j}$, and saturate it with physical sources, in order to obtain the propagator:

$$
\Pi=i \sum_{\alpha \beta, i j, J} \mathcal{S}_{\alpha}^{*} A(J)_{i j}^{-1} P_{i j}(J)_{\alpha \beta} \mathcal{S}_{\beta}^{\prime} .
$$

The propagators provides key ingredients for the understanding the scattering processes of the theory under investigation. In particular, we may analyze more carefully some conditions to guarantee the tree-level unitarity and causality of the Lagrangian model. In general, the poles of the propagators relation for HLG model is

$$
\omega^{2}-Q(k)=0,
$$

where $Q$ may be a polynomial or rational function of the absolute value of the spatial momentum $k$. So, for each propagating pole we will require that $Q(k) \geq 0$, for every momentum $k$, to ensure it is not a tachyon mode. However, at low energies the higher momentum terms becomes less relevant and the dispersion relation takes a simple form

$$
\omega^{2}-c^{2} k^{2}=0
$$

So, for each propagating pole we will require that $c^{2} \geq 0$ to ensure it is not a tachyon mode.

On the other hand, the condition imposed by quantum field theory for the absence of propagating ghosts reads

$$
\Im m \operatorname{Res}\left(\left.\Pi\right|_{\text {pole }}\right)>0 .
$$

Fortunately, with the projection operators formalism we can take advantage from the general decomposition of the degree-of-freedom HLG projection operators,

$$
P_{i j}(J)_{\alpha \beta}=(-1)^{p} \psi_{\alpha}^{(i)} \psi_{\beta}^{(j)},
$$

where $p$ is the parity related to the spin operator, to rewrite the propagator (14) as

$$
\Pi=i(-1)^{P} \sum_{J, i j, f} \mathcal{S}_{i}^{*} A(J, Q)_{i j}^{-1} \mathcal{S}_{j}^{\prime}\left(\omega^{2}-Q(k)\right)^{-1},
$$

where $\mathcal{S}_{j}=\psi_{\alpha}^{(j)} \mathcal{S}^{\alpha}$ and $A(J, Q)_{i j}^{-1}$ are the inverse sub-matrices with the pole extracted. Therefore, the positiveness condition (19), for arbitrary sources, is ensured by the positiveness of the eigenvalues of the $A(J, Q)_{i j}^{-1}$ matrix. Nevertheless, it can be shown, for each case, that these matrices has only one non-vanishing eigenvalue at the pole, which is equal to the trace of $A(J, Q)_{i j}^{-1}$. Therefore, the condition for absence of ghosts for each spin is reduced to:

$$
\left.(-1)^{P} \operatorname{tr} A(J, Q)_{i j}^{-1}\right|_{\text {pole }}>0 .
$$

This technical result converts the task of probing the unitarity, that is in general a time-consuming work, into a straightforward algebraic exercise of analyzing the matrices of coefficients.

\section{DESCRIPTION OF THE LAGRANGIAN MODEL}

Having discussed a general method to obtain the propagator and to analyze the spectral consistency, we may focus our efforts in specific HLG models of our interest. Since one can impose additional symmetries and restrictions to the theory it must be made clear which version of the theory one is referring to. In Hořava's original proposal, probably concerned in maintaining its simplicity, he mainly discussed the version with the detailed balance condition. This condition imposes that the possible spatial metric-dependent terms are of the form $\mathcal{S}_{V}=\int d t d^{3} x \mathcal{N} \sqrt{g} \mathcal{E}^{a b} \mathcal{G}_{a b c d} \mathcal{E}^{c d}$, with $\mathcal{E}^{a b}$ itself following from a variational principle $\sqrt{g} \mathcal{E}^{a b}=\frac{\delta W[g . .]}{\delta g_{a b}}$. Therefore, it implies in a huge reduction in the arbitrary parameters of the model, however it has been shown it becomes very difficult to reconcile this condition with phenomenological considerations [7, 15, 21, 22]. 
Other requirement that significantly alters the structure of HLG models is the projectability condition. It enforces the lapse field to be just a function of time $\mathcal{N}=\mathcal{N}(t)$. Evidently, this avoids Lagrangian terms which are spatial derivatives of $\mathcal{N}$, and thus simplifying the theory. A motivation for imposing this assumption, comes from the possibility of setting $\mathcal{N}=1$ by a gauge fixing. In the following, we shall compare how this condition affects the spectral properties of the model, but without the introduction of auxiliary fields.

Although interesting considerations can be made with both conditions, we shall be mostly concerned with the version where the detailed balance and projectable conditions are neglected. Allowing numerous possible Lagrangian terms may turn the attainment of the propagator a painful task, but with the HLG projection operators this can be done with a systematic and simplified technique. This said, let us enumerate the possible Lagrangian terms compatible with the foliation-preserving diffeomorphisms (Eq. (10).

First, we start with $K_{a b} K^{a b}$ and $K^{2}=\left(g^{a b} K_{a b}\right)^{2}$, which are written in terms of the extrinsic curvature

$$
K_{a b}=\frac{1}{2 \mathcal{N}}\left(\dot{g}_{a b}-\nabla_{a} N_{b}-\nabla_{b} N_{a}\right) .
$$

$K_{a b}$ is covariant under spatial diffeomorphisms and transforms as a scalar under time reparametrization. Here and throughout the manuscript, the dot refers to time derivative and $\nabla_{a}$ is the covariant derivative associated with the three-dimensional metric $g_{a b} . K_{a b} K^{a b}$ and $K^{2}=\left(g^{a b} K_{a b}\right)^{2}$ are referred kinetic terms, since they contain (at most two) time derivatives.

The potential terms do not contain time derivatives. The ones built with the spatial metric $g_{a b}$ have been enumerated in Ref. [31]. Since in three-dimensions, the Riemann tensor is equivalent to the Ricci tensor the independent possible terms up to dimension $[\kappa]^{6}$ are $R^{3}, R R_{b}^{a} R_{a}^{b}, R_{b}^{a} R_{c}^{b} R_{a}^{c}, R \nabla^{2} R, \nabla^{4} R, \nabla_{a} R_{b c} \nabla^{a} R^{b c}, \varepsilon^{a b c} R_{a d} D_{b} R_{c}{ }^{d}, R^{2}, R^{a b} R_{a b}$, $\nabla^{2} R, \varepsilon^{a b c}\left(\Gamma_{a e}^{d} \partial_{b} \Gamma_{c d}^{e}+\frac{2}{3} \Gamma_{a d}^{f} \Gamma_{b e}^{d} \Gamma_{c f}^{e}\right), R$, and 1.

However, the terms above do not exhaust all the possibilities. In fact, an important novelty brought by Blas, Pujolás, and Sibiryakov in Refs. [8, 9] was realizing that terms involving the object $\mathcal{A}_{a}=\mathcal{N}^{-1} \partial_{a} \mathcal{N}$ should be included since it is invariant under foliation-preserving diffeomorphism. The terms mixing $\mathcal{A}_{a}$ with the extrinsic curvature $K_{a b}$ with dimension $[\kappa]^{6}$ or less are $K^{a b} \mathcal{A}_{a} \mathcal{A}_{b}, K^{a b} \nabla_{a} \mathcal{A}_{b}$, and $K \nabla_{a} \mathcal{A}^{a}$. They have the property to be odd under T and CPT transformations. And the list of potential terms built with $\mathcal{A}_{a}$ reads $\mathcal{A}_{a} \mathcal{A}^{a}, \mathcal{A}_{a} \nabla^{2} \mathcal{A}^{a},\left(\mathcal{A}_{a} \mathcal{A}^{a}\right)^{2}, \mathcal{A}_{a} \mathcal{A}_{b} R^{a b}, \mathcal{A}_{a} \nabla^{4} \mathcal{A}^{a}$, $\left(\mathcal{A}_{a} \mathcal{A}^{a}\right)^{3}, \mathcal{A}_{a} \mathcal{A}^{a} \mathcal{A}_{b} \mathcal{A}_{c} R^{b c} \ldots$ Notably, with this improvement it has been argued that HLG does not run into problems with strong coupling and could be a phenomenologically viable model $[8,9]$.

After this brief consideration on the development of HLGs (for a deeper discussion we address the reader to Refs. $[2,7[9,15,27,31,33,37])$, we set up a general HLG model only with the terms that affect the propagator,

$$
S_{H L}=\int d t d^{3} x \sqrt{g} N\left(\alpha K_{a b} K^{a b}+\beta K^{2}+\mathcal{L}_{g}+\mathcal{L}_{\mathcal{A}}\right),
$$

where the potential terms related only to the spatial metric are

$$
\mathcal{L}_{g}=a_{1} R+b_{1} R_{a b} R^{a b}+c_{1} R^{2}+a_{2} \Delta R+b_{2} R_{a b} \Delta R^{a b}+c_{2} R \Delta R+a_{3} \Delta^{2} R+\mu \mathcal{L}_{C S}+\lambda \mathcal{L}_{R C},
$$

where the Chern-Simons like term reads

$$
\mathcal{L}_{C S}=\varepsilon^{a b c}\left(\Gamma_{a e}^{d} \partial_{b} \Gamma_{c d}^{e}+\frac{2}{3} \Gamma_{a d}^{f} \Gamma_{b e}^{d} \Gamma_{c f}^{e}\right)
$$

and the Ricci-Cotton term is cast as

$$
\mathcal{L}_{R C}=\varepsilon^{a b c} R_{a d} D_{b} R_{c}^{d} .
$$

The Lagrangian with the terms related with $\mathcal{A}_{a}$ reads,

$$
\begin{aligned}
\mathcal{L}_{\mathcal{A}} & =r K^{a b} \nabla_{a} \mathcal{A}_{b}+s K \nabla_{a} \mathcal{A}^{a}+u_{1} R \nabla_{a} \mathcal{A}^{a}+u_{2} \Delta R \nabla_{a} \mathcal{A}^{a} \\
& +\eta_{1} \mathcal{A}_{a} \mathcal{A}^{a}+\eta_{2} \mathcal{A}_{a} \Delta \mathcal{A}^{a}+\eta_{3} \mathcal{A}_{a} \Delta^{2} \mathcal{A}^{a} .
\end{aligned}
$$

We should emphasize that, at this point, we shall adopt the posture of not avoiding the discussion of the terms that violate $\mathrm{P}, \mathrm{T}$ or CPT symmetry. Up until now, we have no fundamental reason for leaving them out and it is our interest to understand how they affect the propagating modes dispersion relations. 


\section{ATTAINMENT OF THE PROPAGATORS AND SPECTRAL ANALYSIS FOR THE GENERAL HOŘAVA-LIFSHITZ GRAVITY MODEL}

Having established the model of our interest, we may work in the wave operator defined by Eq. (24). After some algebraic calculations (including partial integrations), it is possible to bring the quadratic Lagrangian for the action (24) into the form of the Eq. (12). With this procedure we determine the corresponding wave operator. In the sequel, we perform the wave operator decomposition in terms of the HLG projection operators, as indicated by Eq. (13), aided by the relations developed in the Appendix B.

Some remarks are in order. In the attainment of the spin- 1 matrix of coefficients one realizes that there is no need for decomposing the propagating modes its degree-of-freedom, since it appears no parity violating contribution in this sector. For simplicity, we write them in terms of the parity-preserving operators (7b). On the other hand, the Chern-Simons and Ricci-Cotton parity breaking terms (Eqs. (25b) and (25c), respectively) imply the violation of parity in the spin-2 sector. It forces us to use the degree-of-freedom operators (10b) in this sector. With this in mind, we write out the coefficients matrices of the wave operator expansion,

$$
\begin{aligned}
& a(2)=\left(\begin{array}{cc}
\frac{\alpha}{2} \omega^{2}-\frac{1}{2}\left(a-b k^{2}\right) k^{2} & -i k^{2}\left(\mu+\frac{1}{2} \lambda k^{2}\right) \sqrt{k^{2}} \\
i k^{2}\left(\mu+\frac{1}{2} \lambda k^{2}\right) \sqrt{k^{2}} & \frac{\alpha}{2} \omega^{2}-\frac{1}{2}\left(a-b k^{2}\right) k^{2}
\end{array}\right) \\
& a(1)=\left(\begin{array}{cc}
\frac{\alpha}{2} \omega^{2} & -\alpha \omega \frac{\sqrt{k^{2}}}{\sqrt{2}} \\
-\alpha \omega \frac{\sqrt{k^{2}}}{\sqrt{2}} & \alpha k^{2}
\end{array}\right), \\
& a(0)=\left(\begin{array}{cccc}
\left(\frac{\alpha}{2}+\beta\right) \omega^{2}+\frac{1}{2} A k^{2} & \frac{\beta}{\sqrt{2}} \omega^{2} & -\beta \omega \sqrt{2 k^{2}} & \sqrt{2}\left(a-u k^{2}\right) k^{2}+\frac{1}{\sqrt{2}} s i \omega k^{2} \\
\frac{\beta}{\sqrt{2}} \omega^{2} & \frac{1}{2}(\alpha+\beta) \omega^{2} & -(\alpha+\beta) \omega \sqrt{k^{2}} & \frac{1}{2}(r+s) i \omega k^{2} \\
-\beta \omega \sqrt{2 k^{2}} & -(\alpha+\beta) \omega \sqrt{k^{2}} & 2(\alpha+\beta) k^{2} & -(r+s) i k^{2} \sqrt{k^{2}} \\
\sqrt{2}\left(a-u k^{2}\right) k^{2}-\frac{1}{\sqrt{2}} s i \omega k^{2} & -\frac{1}{2}(r+s) i \omega k^{2} & (r+s) i k^{2} \sqrt{k^{2}} & 2 \eta k^{2}
\end{array}\right),
\end{aligned}
$$

where we have redefined some coefficients to avoid cluttering the notation:

$$
\begin{aligned}
a & =a_{1}-a_{2} k^{2}+a_{3} k^{4} \\
b & =b_{1}-b_{2} k^{2} \\
c & =c_{1}-c_{2} k^{2} \\
u & =u_{1}-u_{2} k^{2} \\
\eta & =\eta_{1}-\eta_{2} k^{2}+\eta_{3} k^{4} \\
A & =a+(3 b+8 c) k^{2} .
\end{aligned}
$$

As mentioned in Sec. II] the degeneracy structure of the matrices (26a)- 26c) brings us information about the gauge symmetries and the source constraints of the model. In particular, the spin-1 matrix is degenerate and the $2^{\text {nd }}$ and $3^{r d}$ rows of the spin-0 matrix are proportional. Adding together the result of equation (15b) for these two matrices, one obtains

$$
\frac{\omega}{2} \mathcal{S}_{b}+k_{a} \mathcal{S}_{a b}=0
$$

For a consistency test, one may verify that for the Einstein-Hilbert gravity (EHG) $\left(\alpha=-\beta=a_{1}=\frac{1}{16 \pi G}\right.$ and all other coefficients vanishing) there appears another symmetry in the spin-0 matrix: the $3^{\text {rd }}$ and $4^{\text {th }}$ columns and rows will become proportionate. It will give rise to another constraint among the sources

$$
\omega \mathcal{S}+k_{a} \mathcal{S}_{a}=0 \quad \text { (only for E-H). }
$$

These results are compatible with the relativistic source constraint $k_{\mu} \mathcal{S}^{\mu \nu}=0(\mu, \nu=0,1,2,3)$ if one takes into account that: (i) the contributions of $h^{0 a}$ and $h^{a 0}$ are encoded in $N^{a}$; it generates a factor of 2 in the matrix decomposition, which implies in a $\frac{1}{2}$-factor in the source $\mathcal{S}_{a}$ and (ii) the weak field expansion $\mathcal{N}=1+g$, along with the definition in the ADM decomposition of ${ }^{4} g_{00}$ implies that $h^{00}$ is identified with $2 n$; this also brings a $\frac{1}{2}$-factor associated with the source $\mathcal{S}$.

With these matrices coefficients, we may follow the analysis of the spectral consistency as proposed in section $\amalg$ For the sake of clarity, we separated the discussion for each spin mode. 


\section{A. Spin-2 Sector}

Let us analyze is the spin-2 mode of the graviton. The matrix $a(2)$ from equation (26a) is non-degenerate. To obtain the propagator (14) its inverse may be readily obtained

$$
a(2)^{-1}=\frac{1}{D_{(2)}}\left(\begin{array}{cc}
\frac{\alpha}{2} \omega^{2}-\frac{1}{2}\left(a-b k^{2}\right) k^{2} & -i\left(\mu+\frac{1}{2} \lambda k^{2}\right) k^{2} \sqrt{k^{2}} \\
i\left(\mu+\frac{1}{2} \lambda k^{2}\right) k^{2} \sqrt{k^{2}} & \frac{\alpha}{2} \omega^{2}-\frac{1}{2}\left(a-b k^{2}\right) k^{2}
\end{array}\right)
$$

where the denominator $D_{(2)}=\left(\frac{\alpha}{2} \omega^{2}-\frac{1}{2}\left(a-b k^{2}\right) k^{2}\right)^{2}-\left(\mu+\frac{1}{2} \lambda k^{2}\right)^{2} k^{6}$ has two poles in the energy. This is an interesting feature of the parity breaking terms: they allow each spin component to propagate independently, with different dispersion relations

$$
\omega_{ \pm}^{2}=\frac{1}{\alpha}\left(a-b k^{2}\right) k^{2} \pm \frac{2}{\alpha}\left(\mu+\frac{1}{2} \lambda k^{2}\right) k^{2} \sqrt{k^{2}} .
$$

Using the posture of requiring a positive speed for every propagating mode, one must ensure that

$$
c_{(2)}^{2}=\frac{a_{1}}{\alpha}>0
$$

The condition for the absence of ghosts must be made for each pole $\omega_{ \pm}^{2}=Q_{ \pm}(k)$. In either cases, (22) implies that $1 / \alpha>0$, so the ghost and tachyon free conditions are given by

$$
\text { Spin-2: } a_{1}>0 ; \alpha>0 \text {. }
$$

From this analysis we conclude that the Chern-Simons and Ricci-Cotton terms, which are related with the parameters $\mu$ and $\lambda$, do not interfere in the unitary relations as its related terms in the three-dimensional topological gravity.

\section{B. Spin-1 Sector}

Since the spin-1 matrix is degenerate, we shall use the prescription of the equation (16). A largest non-degenerate matrix can be obtained by deleting its $2^{\text {nd }}$ columns and rows. Its inverse is given by

$$
A(1)^{-1}=\frac{2}{\alpha \omega^{2}}
$$

This mode poses us with a strange pole $\omega^{2}=0$. However, (34) is related to the projection operator $P_{11}^{h h}(1)=$ $\frac{1}{2}\left(\theta_{a c} \omega_{b d}+\theta_{b c} \omega_{a d}+\theta_{a d} \omega_{b c}+\theta_{b d} \omega_{a c}\right)$, which implies that the spatial momentum $k_{a}$ is contracted with the source $\mathcal{S}^{a b}$ in the expression (16). In this way, using the source constraint (28), one verifies that the residue at the pole of the propagator saturated with physical sources vanishes identically. We must therefore understand this pole as a non-propagating mode.

\section{Spin-0 Sector}

We left for last the spin-0 sector, which brings the most interesting discussions in the literature. It is a natural consequence of the lost of symmetry that new propagating modes appear and this is reflected in this sector. However, it is of a major importance to verify the spectral consistency of this mode. In fact, the EHG augmented with relativistic higher-derivative corrections has been shown to be renormalizable [55], but exactly the 4D spin 0 mode yields a ghost excitation which jeopardizes unitarity of S-matrix and its possibility to become a consistent quantum model for gravitation.

The second and third columns of the matrix $a(0)$ (equation (26c)) are degenerate, due to a residual gauge symmetry. The inverse of $A(0)$ is cast in the Appendix $\mathrm{A}$ and stems a dispersion relation,

$$
\omega^{2}+\frac{\left(\frac{1}{2} A \eta-\left(a-u k^{2}\right)^{2}\right)(\alpha+\beta)-\frac{1}{8} A k^{2}(r+s)^{2}}{\frac{1}{2} \alpha \eta(\alpha+3 \beta)-\frac{1}{8}\left(\alpha\left((r+s)^{2}+2 s^{2}\right)+2 \beta r^{2}\right) k^{2}} k^{2}=0
$$


This great number of arbitrary parameters makes the spectral analysis a complicated tasks and many intriguing situation occurs. In the special case where $\eta=u=0$, and $r=-s \neq 0$ we obtain the dispersion relation $\omega^{2}+4 \frac{a^{2}}{r^{2}}=0$, which is a constant negative energy solution.

If the CPT violating terms are absent $r=s=0$, we have no great difficulty to proceed. In this situation,

$$
A(0)^{-1}=\frac{1}{D_{(0)}}\left(\begin{array}{ccc}
2 \eta(\alpha+\beta) & -2 \sqrt{2} \eta \beta & -\sqrt{2} a(\alpha+\beta) \\
-2 \sqrt{2} \eta \beta & \frac{2 \eta(\alpha+2 \beta) \omega^{2}-\left(4 a^{2}-2 A \eta\right) k^{2}}{\omega^{2}} & 2 a \beta \\
-a \sqrt{2}(\alpha+\beta) & 2 a \beta & \frac{(\alpha+3 \beta) \alpha \omega^{2}+(\alpha+\beta) A k^{2}}{2 k^{2}}
\end{array}\right),
$$

with $D_{(0)}=\eta(\alpha+3 \beta) \alpha \omega^{2}-\left(2 a^{2}-A \eta\right)(\alpha+\beta) k^{2}$ and the dispersion relation is given by

$$
\omega^{2}-\frac{\left(2 a^{2}-A \eta\right)}{\eta} \frac{(\alpha+\beta)}{(\alpha+3 \beta) \alpha} k^{2}=0
$$

which in general is a rational function of the spatial momentum $k^{2}$. It recovers the dispersion relation obtained in the reference [7].

For small values of momentum the positivity of the propagation speed for this mode reads,

$$
c_{(0)}^{2}=\frac{a_{1}\left(2 a_{1}-\eta_{1}\right)}{\eta_{1}} \frac{(\alpha+\beta)}{(\alpha+3 \beta) \alpha}>0 .
$$

To obtain the ghost-free condition, one ought to verify the residue of the matrix at the pole. Replacing the dispersion relation (37) and after a inspection (in a CAS, for example) one verifies that $\left.A(0)^{-1}\right|_{\text {pole }}$ has only one non-vanishing eigenvalue, which is equal to its trace, so the expression (22) is valid,

$$
\left.\operatorname{tr} A(0)^{-1}\right|_{\text {pole }}=\frac{\left(a^{2}+2 \eta^{2}\right)(\alpha+\beta)^{2}+4 \beta^{2} \eta^{2}}{\alpha \eta^{2}(\alpha+\beta)(\alpha+3 \beta)} .
$$

In this way, the condition for absence of ghosts can be stated as

$$
\alpha(\alpha+\beta)(\alpha+3 \beta)>0 \text {. }
$$

Combining the results (38) and (40) with the spin-2 condition (33) one must impose that

$$
\text { Spin 0 : }(\alpha+\beta)(\alpha+3 \beta)>0 ; \eta_{1}\left(2 a_{1}-\eta_{1}\right)>0,
$$

for the absence of ghost and tachyon in this sector.

For comparison, let us come back and analyze how the projectability condition affects the matrix structures and thus the propagating modes. Imposing the restriction that the lapse field does not depend on the space variables $\mathcal{N}=\mathcal{N}(t)$ implies that the terms in the quadratic Lagrangian containing the perturbation of the lapse $n$ are total derivatives. Thus, the fourth column and row of the spin 0 coefficient matrix vanishes identically,

$$
a(0)_{p}=\left(\begin{array}{cccc}
\left(\frac{\alpha}{2}+\beta\right) \omega^{2}+\frac{1}{2} A k^{2} & \frac{\beta}{\sqrt{2}} \omega^{2} & -\beta \omega \sqrt{2 k^{2}} & 0 \\
\frac{\beta}{\sqrt{2}} \omega^{2} & \frac{1}{2}(\alpha+\beta) \omega^{2} & -(\alpha+\beta) \omega \sqrt{k^{2}} & 0 \\
-\beta \omega \sqrt{2 k^{2}} & -(\alpha+\beta) \omega \sqrt{k^{2}} & 2(\alpha+\beta) k^{2} & 0 \\
0 & 0 & 0 & 0
\end{array}\right) .
$$

With this simplification, one readily realizes that $a(0)_{p}$ is doubly degenerate: the column 4 is null and the columns 2 and 3 are proportional. A largest non-degenerate matrix of $a(0)_{p}$ is obtained by deleting the $3^{r d}$ and $4^{\text {th }}$ columns and rows,

$$
A(0)_{p}=\left(\begin{array}{cc}
\left(\frac{\alpha}{2}+\beta\right) \omega^{2}+\frac{1}{2} A k^{2} & \frac{\beta}{\sqrt{2}} \omega^{2} \\
\frac{\beta}{\sqrt{2}} \omega^{2} & \frac{1}{2}(\alpha+\beta) \omega^{2}
\end{array}\right)
$$

Its inverse is given by

$$
A(0)_{p}^{-1}=\frac{4}{D_{(0) p} \omega^{2}}\left(\begin{array}{cc}
\frac{1}{2}(\alpha+\beta) \omega^{2} & -\frac{\beta}{\sqrt{2}} \omega^{2} \\
-\frac{\beta}{\sqrt{2}} \omega^{2} & \left(\frac{\alpha}{2}+\beta\right) \omega^{2}+\frac{1}{2} A k^{2}
\end{array}\right)
$$


with

$$
D_{(0) p}=(\alpha+3 \beta) \alpha \omega^{2}+(\alpha+\beta) A k^{2} .
$$

providing the dispersion relation for this propagating mode. To ensure that this mode is not a tachyonic excitation we must impose

$$
c_{(0) p}^{2}=-\frac{(\alpha+\beta) a_{1}}{(\alpha+3 \beta) \alpha}>0 .
$$

It can be explicitly verified that $\left.\operatorname{Res}\left\{A(0)^{-1}\right\}\right|_{\text {pole }}$ is degenerate, in such a way that its only non-vanishing eigenvalue matches its trace. So one is enforced to impose that

$$
\operatorname{tr}\left(\left.\operatorname{Res}\left\{A(0)^{-1}\right\}\right|_{\text {pole }}\right)=\frac{2(\alpha+\beta)^{2}+4 \beta^{2}}{\alpha(\alpha+\beta)(\alpha+3 \beta)}>0
$$

The only way to reconcile the conditions (46) and (47) would be to impose $a_{1}<0$; but, this contradicts the unitarity condition for the spin 2 sector. Therefore, we may infer that the HLG with the projectable condition is not compatible with perturbative unitarity at the tree-level approximation.

An alternative to circumvent this issue is to enhance the model with an Abelian gauge symmetry [3, 4, 12, 30], in order to inhibit the propagation of the spin-0 mode. This is possible with the introduction of a gauge field $A$ and an auxiliary scalar field $\nu$, known as the Newtonian prepotential. This approach yields a model with the same number of degrees-of-freedom as the EHG.

\section{CONCLUDING ASSESSMENT}

In this manuscript, we have built a set of orthonormal projection operators suitable for non-relativistic theories of gravity, including the ones with parity violating terms. The advantage of this construction for the attainment of the propagator and the spectral analysis, which is in general a time-consuming task, is that it can be done in a systematic and simplified way, including the handling of the gauge symmetries of the model. With this methodology, we were able to determine conditions over the coefficients of general Hořava-Lifshitz gravity (HLG) in order to restrict the propagation of tachyons and ghosts.

With the general result of the propagators (Eqs. (30), (34), and (36)), we may contemplate some results in a low-energy regime of the theory, where the terms depending on higher spatial momentum become less relevant. In such situation, the general action of the nonprojectable HLG (Eq. (24) ) may be simplified to

$$
S_{H L G}=\int d t d^{3} x \sqrt{g} \mathcal{N}\left(\alpha K_{a b} K^{a b}+\beta K^{2}+a R+\eta \mathcal{A}_{a} \mathcal{A}^{a}\right) .
$$

and the inverse of the coefficient matrices of the propagator for this simplified model are cast as

$$
\begin{gathered}
A(0)_{H L G}^{-1}=\frac{1}{D_{(0)}}\left(\begin{array}{ccc}
2 \eta(\alpha+\beta) & -2 \sqrt{2} \eta \beta & -\sqrt{2} a(\alpha+\beta) \\
-2 \sqrt{2} \eta \beta & \frac{2 \eta(\alpha+2 \beta) \omega^{2}-2 a(2 a-\eta) k^{2}}{\omega^{2}} & 2 a \beta \\
-\sqrt{2} a(\alpha+\beta) & 2 a \beta & \frac{(\alpha+3 \beta) \alpha \omega^{2}+(\alpha+\beta) a k^{2}}{2 k^{2}}
\end{array}\right), \\
A(1)_{H L G}^{-1}=\frac{2}{\alpha \omega^{2}}, \quad a(2)_{H L G}^{-1}=\frac{2}{\alpha \omega^{2}-a k^{2}},
\end{gathered}
$$

with $D_{(0)}=\eta(\alpha+3 \beta) \alpha \omega^{2}-a(2 a-\eta)(\alpha+\beta) k^{2}$.

It is instructive to compare the propagators of the HLG (Eqs. (49a) and (49b) with the Einstein-Hilbert gravity (EHG) propagators in ADM coordinates with HLG projection operators. They may be written as

$$
A(0)_{E H G}^{-1}=\frac{1}{\alpha k^{2}}\left(\begin{array}{cc}
0 & \frac{1}{\sqrt{2}} \\
\frac{1}{\sqrt{2}} & \frac{1}{4 k^{2}}\left(\omega^{2}-k^{2}\right)
\end{array}\right),
$$




$$
A(1)_{E H G}^{-1}=\frac{2}{\alpha \omega^{2}}, \quad a(2)_{E H G}^{-1}=\frac{2}{\alpha\left(\omega^{2}-k^{2}\right)} .
$$

By a quick glance at these matrices of coefficients one may set up comparisons between both models. Analyzing the pole structure of the propagators, we may conclude that the spin- 2 massless mode propagate in both models, but with different speed of propagation. This corresponds to the usual helicity- 2 graviton mode of the relativistic model. Both models coincide by not having dynamical poles in the spin-1 sector. However, HLG has a propagating pole the spin-0 sector, which is absent in the EHG.

This new degree of freedom may lead to odd effects. For example, the helicity-0 mode of massless limit of Pauli-Fierz gravity (PFG) generates a factor $\frac{3}{4}$ in the bending of the light by the Sun, if one scales the coupling constant of both PFG and EHG such that the Newtonian limit holds [56 60] (see Ref. [61 for a recent review on the subject). This effect can be explained by the different way that the helicity- 2 and helicity- 0 modes couple to scalar and vectorial matter sources. One should be aware that this apparent paradox, known as vDVZ discontinuity, appears only in the perturbative approach of the linearized theory.

Now, we are ready to compare the HLG and EHG in a situation of a point mass static source coupled to relativistic matter sources. The static point-like source can be described by $\mathcal{S}=M \delta(\omega), \mathcal{S}_{a}=\mathcal{S}_{a b}=0$. The propagator amplitude $\Pi$ for this source coupled to arbitrary sources $\left\{\mathcal{S}^{\prime}, \mathcal{S}_{a}^{\prime}, \mathcal{S}_{a b}^{\prime}\right\}$ is

$$
\begin{aligned}
-i \Pi_{H L G} & =M \delta(\omega) \frac{1}{(2 a-\eta) k^{2}}\left\{\left(\theta_{a b}-\frac{2 \beta}{(\alpha+\beta)} \omega_{a b}\right) \mathcal{S}_{a b}^{\prime}-\frac{1}{2} \mathcal{S}^{\prime}\right\}, \\
-i \Pi_{E H G} & =M \delta(\omega) \frac{1}{2 \alpha k^{2}}\left\{\theta_{a b} \mathcal{S}_{a b}^{\prime}-\frac{1}{2} \mathcal{S}^{\prime}\right\}
\end{aligned}
$$

respectively for HLG and EHG. In the particular case of the Newtonian test with a point-like source of mass $M^{\prime}$ separated by the distance of $R$, the energy of interaction is given by

$$
E_{H L G}=-\frac{1}{2(2 a-\eta)} \frac{M M^{\prime}}{R}, \quad E_{E H G}=-\frac{1}{4 \alpha} \frac{M M^{\prime}}{R},
$$

respectively for HLG and EHG.

On the other hand, the relativistic electromagnetic matter should be dealt more carefully than just a scalar matter, for which $\mathcal{S}_{\mu}^{\mu}=0$. In terms of the ADM-components the traceleness of the electromagnetic tensor reads,

$$
\frac{1}{2} \mathcal{S}_{E M}^{\prime}+\delta_{a b} \mathcal{S}_{E M}^{\prime a b}=0
$$

Using the identity (53) together with the source conservation constraint (28) one can show that the interaction energy of the point mass with the light beam exceeds the value of Eq. (52) by a factor of two for both theories (replacing mass with total energy). This implies that these experiments with static sources cannot distinguish the EHG from the nonprojectable HLG at the tree-level order of perturbation, since both are scaled by multiplicative constant.

In this vein, one may look foward for a dynamical experiment that may unveil the effects of the extra scalar mode. An interesting possibility is to set up the test of gravitational pulsar experiment. It consists of a spherically symmetric harmonically pulsating mass distribution. In fact, according to the Birkhoff theorem, the EHG radiation from any spherically symmetric source is zero [62]. Looking to the propagators coefficients of the EHG (Eqs. (50a)-(50b)) one may verify that it is indeed the case, since the propagating pole of the EHG is associated with a transverse operator $P(2)$ that vanishes when contracted with a spherically symmetric source. The propagator structure of HLG leads to a different situation, because it has non-vanishing coefficients with propagating pole associated with longitudinal operators. In this case only the spin- 0 mode carries radiating energy and, hence, the Birkhoff theorem is not valid in the nonprojectable HLG.

Our efforts here do not contemplate the incorporation of the cosmological constant in connection with the spectral analysis of the modes present in HLG. We understand that this is a fairly interesting issue, which may motivate further investigation. Indeed, in the recent paper of Ref. [26], the authors pursue a stimulating discussion on the stability of HLG black holes in connection with the AdS/CFT correspondence and possible applications of the holographic principle. In view of that, we shall be going further to endeavor a study of the consequences of the cosmological constant in the frame of the approach we report here.

Also, the coupling of Lifshitz fermions to HLG could be an interesting matter in connection with our present investigation, since torsion degrees of freedom may be excited and their incorporation shall yield new interesting technical aspects in our treatment. The extension of our method to include torsion in the HLG frame is also motivated by the need to study the possible production and the consequent decay of massive gravitons at the TeV scale to finally compare with the results worked out in previous Lorentz-preserving quantum gravity models [63 69]. 


\section{ACKNOWLEDGMENTS:}

The authors express their gratitude to Prof. A. J. Accioly for helpful comments and suggestions. J. L. L. Morais, E. Scatena, and R. Turcati are also acknowledged for the supporting discussions and G. N. Bremm for the careful reading of the manuscript. Thanks are also due to CNPq-Brazil and CAPES-Brazil for the financial support.

\section{Appendix A: Inverse of $A(0)$ Matrix}

The inverse of $A(0)$ matrix that is obtained by deleting the $3^{\text {rd }}$ row and column of $a(0)$ (equation (26c)) is given by:

$$
A(0)^{-1}=\frac{1}{D_{0}}\left(\begin{array}{ccc}
A_{11}^{(0)} & A_{12}^{(0)} & A_{13}^{(0)} \\
A_{12}^{(0) *} & A_{22}^{(0)} & A_{23}^{(0)} \\
A_{13}^{(0) *} & A_{23}^{(0) *} & A_{33}^{(0)}
\end{array}\right),
$$

where

$$
\begin{aligned}
D_{0} & =\left\{\left[\frac{1}{2} \alpha \eta(\alpha+3 \beta)-\frac{1}{8} k^{2}\left(\alpha\left((r+s)^{2}+2 s^{2}\right)+2 \beta r^{2}\right)\right] \omega^{2}\right. \\
& \left.+\left[\left(\frac{1}{2} A \eta-\left(a-u k^{2}\right)^{2}\right)(\alpha+\beta)-\frac{1}{8} A k^{2}(r+s)^{2}\right] k^{2}\right\} k^{2} \omega^{2} \\
A_{11}^{(0)} & =\left((\alpha+\beta) \eta-\frac{1}{4}(r+s)^{2} k^{2}\right) \omega^{2} k^{2} \\
A_{12}^{(0)} & =-\sqrt{2} \beta \eta \omega^{2} k^{2}-\frac{i}{\sqrt{2}}(r+s)\left(a-u k^{2}+\frac{1}{2} s i \omega\right) \omega k^{4} \\
A_{13}^{(0)} & =-\left(\frac{1}{2 \sqrt{2}} i(s \alpha-r \beta) \omega+\frac{1}{\sqrt{2}}\left(a-u k^{2}\right)(\alpha+\beta)\right) \omega^{2} k^{2} \\
A_{22}^{(0)} & =(\alpha+2 \beta) \eta \omega^{2} k^{2}-\left(\frac{1}{2} s^{2} \omega^{2}+2\left(a-u k^{2}\right)^{2}-A \eta\right) k^{4} \\
A_{23}^{(0)} & =-\frac{1}{4} i((r+s) \alpha+2 r \beta) k^{2} \omega^{3}+\left(a-u k^{2}\right) \beta k^{2} \omega^{2}-\frac{1}{4} i A k^{4}(r+s) \omega \\
A_{33}^{(0)} & =\frac{1}{4}\left((\alpha+3 \beta) \alpha \omega^{2}+A(\alpha+\beta) k^{2}\right) \omega^{2}
\end{aligned}
$$

\section{Appendix B: Hořava-Lifshitz Gravity Projection Operators and Tensorial Relations}

In order to facilitate further use of the set of the degree-of-freedom and the parity preserving HLG projection operators we write out some useful identities satisfied by them. To avoid cluttering the notation, some indices of operators may be omitted.

\section{Vector field operators: $N^{a}-N^{a}$}

Identities Among the Operators

$$
P^{N N}(1)_{a b}=P_{33}^{N N}\left(1^{++}\right)_{a b}+P_{44}^{N N}\left(1^{--}\right)_{a b}
$$

Tensorial Identities

$$
\begin{aligned}
\delta_{a b} & =P_{33}^{N N}(0)_{a b}+P^{N N}(1)_{a b} \\
k_{a} k_{b} & =k^{2} P_{33}^{N N}(0)_{a b} \\
\varepsilon_{a b c} k^{c} & =\sqrt{k^{2}}\left(P_{12}^{N N}\left(1^{++}\right)_{a b}-P_{21}^{N N}\left(1^{--}\right)_{a b}\right)
\end{aligned}
$$




\section{Rank-2 Symmetric Field Operators, $h-h$}

Identities Among the Operators

$$
\begin{aligned}
& P^{h h}(1)_{a b ; c d}=P_{11}^{h h}\left(1^{++}\right)+P_{22}^{h h}\left(1^{--}\right) \\
& P^{h h}(2)_{a b ; c d}=P_{11}^{h h}\left(2^{++}\right)+P_{22}^{h h}\left(2^{--}\right)
\end{aligned}
$$

Tensorial Identities

$$
\begin{aligned}
& \delta_{a b, c d}=\frac{1}{2}\left(\delta_{a c} \delta_{b d}+\delta_{a d} \delta_{b c}\right)=P^{h h}(2)+P^{h h}(1)+P_{11}^{h h}\left(0^{s}\right)+P_{22}^{h h}\left(0^{\omega}\right) \\
& \delta_{a b} \delta_{c d}=2 P_{11}^{h h}\left(0^{s}\right)+\sqrt{2} P_{12}^{h h}\left(0^{s \omega}\right)+\sqrt{2} P_{21}^{h h}\left(0^{\omega s}\right)+P_{22}^{h h}\left(0^{\omega}\right) \\
& k_{a} k_{b} \delta_{c d}+k_{c} k_{d} \delta_{a b}=\sqrt{2} k^{2}\left(P_{12}^{h h}\left(0^{s \omega}\right)+P_{21}^{h h}\left(0^{\omega s}\right)\right)+2 k^{2} P_{22}^{h h}\left(0^{\omega}\right) \\
& k_{a} k_{c} \delta_{b d}+k_{a} k_{d} \delta_{b c}+k_{b} k_{c} \delta_{a d}+k_{b} k_{d} \delta_{a c}=2 k^{2} P^{h h}(1)+4 k^{2} P_{22}^{h h}\left(0^{\omega}\right) \\
& k_{a} k_{b} k_{c} k_{d}=k^{4} P_{22}^{h h}\left(0^{\omega}\right) \\
& \left(\varepsilon_{e c a} \delta_{b d}+\varepsilon_{e c b} \delta_{a d}+\varepsilon_{e d a} \delta_{b c}+\varepsilon_{e d b} \delta_{a c}\right) k^{e}=2 \sqrt{k^{2}}\left(2 P_{12}^{h h}\left(2^{-+}\right)-2 P_{21}^{h h}\left(2^{+-}\right)-P_{12}^{h h}\left(1^{+-}\right)+P_{21}^{h h}\left(1^{-+}\right)\right)(\mathrm{B} \\
& \left(\varepsilon_{e c a} k_{b} k_{d}+\varepsilon_{e c b} k_{a} k_{d}+\varepsilon_{e d a} k_{b} k_{c}+\varepsilon_{e d b} k_{a} k_{c}\right) k^{e}=2 k^{2} \sqrt{k^{2}}\left(-P_{12}^{h h}\left(1^{+-}\right)+P_{21}^{h h}\left(1^{-+}\right)\right)
\end{aligned}
$$

\section{Graviton-Vector Operator, $h-N$}

Tensorial Identities

$$
\begin{aligned}
& P^{h N}\left(1^{+}\right)_{a b ; c}=P_{13}^{h N}\left(1^{++}\right)+P_{24}^{h N}\left(1^{--}\right) \\
& \delta_{a b} k_{c}=\sqrt{2 k^{2}} P_{13}^{h N}\left(0^{s}\right)+\sqrt{k^{2}} P_{23}^{h N}\left(0^{++}\right) \\
& \frac{1}{2}\left(k_{a} \delta_{c b}+k_{b} \delta_{c a}\right)=\frac{\sqrt{k^{2}}}{\sqrt{2}}\left(P_{13}^{h N}\left(1^{++}\right)+P_{24}^{h N}\left(1^{--}\right)\right)+\sqrt{k^{2}} P_{23}^{h N}\left(0^{++}\right)
\end{aligned}
$$

\section{Graviton-Vector Operator, $N-h$}

Tensorial Identities

$$
\begin{aligned}
& P^{N h}\left(1^{+}\right)_{a ; b c}=P_{31}^{N h}\left(1^{++}\right)+P_{42}^{N h}\left(1^{--}\right) \\
& k_{a} \delta_{b c}=\sqrt{2 k^{2}} P_{31}^{N h}\left(0^{s}\right)+\sqrt{k^{2}} P_{32}^{N h}\left(0^{\omega}\right) \\
& \frac{1}{2}\left(k_{b} \delta_{a c}+k_{c} \delta_{a b}\right)=\frac{\sqrt{k^{2}}}{\sqrt{2}}\left(P_{31}^{N h}\left(1^{++}\right)+P_{42}^{N h}\left(1^{--}\right)\right)+\sqrt{k^{2}} P_{32}^{N h}\left(0^{\omega}\right)
\end{aligned}
$$

[1] P. Horava, Membranes at Quantum Criticality, JHEP 0903, 020 (2009) arXiv:0812.4287 [hep-th]].

[2] P. Horava, Quantum Gravity at a Lifshitz Point, Phys. Rev. D 79, 084008 (2009) arXiv:0901.3775 [hep-th]].

[3] P. Horava, C. M. Melby-Thompson, General Covariance in Quantum Gravity at a Lifshitz Point, Phys. Rev. D82, 064027 (2010). arXiv:1007.2410 [hep-th]]

[4] P. Horava, General Covariance in Gravity at a Lifshitz Point, Class. Quant. Grav. 28, 114012 (2011). arXiv:1101.1081 [hep-th]].

[5] F. S. Bemfica and M. Gomes, Fourth order spatial derivative gravity, Phys. Rev. D 84, 084022 (2011) arXiv:1108.5979 [hep-th]].

[6] F. S. Bemfica and M. Gomes, Propagator in the Horava-Lifshitz gravity, arXiv:1111.5779 [hep-th].

[7] D. Blas, O. Pujolàs and S. Sibiryakov, On the Extra Mode and Inconsistency of Horava Gravity, JHEP 0910, 029 (2009) arXiv:0906.3046 [hep-th]].

[8] D. Blas, O. Pujolàs and S. Sibiryakov, Consistent Extension Of Horava Gravity, Phys. Rev. Lett. 104, 181302 (2010) arXiv:0909.3525 [hep-th]]. 
[9] D. Blas, O. Pujolàs and S. Sibiryakov, Models of non-relativistic quantum gravity: The Good, the bad and the healthy, JHEP 1104, 018 (2011). arXiv:1007.3503 [hep-th]].

[10] D. Blas, H. Sanctuary, Gravitational Radiation in Horava Gravity, arXiv:1105.5149 [gr-qc]].

[11] C. Bogdanos and E. N. Saridakis, Perturbative instabilities in Horava gravity, Class. Quant. Grav. 27, 075005 (2010) arXiv:0907.1636 [hep-th]].

[12] A. Borzou, K. Lin, A. Wang, Detailed balance condition and ultraviolet stability of scalar field in Horava-Lifshitz gravity, JCAP 1105, 006 (2011). arXiv:1103.4366 [hep-th]].

[13] R.-G. Cai, B. Hu, H.-B. Zhang, Scalar graviton in the healthy extension of Hořava-Lifshitz theory, Phys. Rev. D83, 084009 (2011). arXiv:1008.5048 [hep-th]].

[14] G. Calcagni, Detailed balance in Horava-Lifshitz gravity, Phys. Rev. D81, 044006 (2010). arXiv:0905.3740 [hep-th]].

[15] C. Charmousis, G. Niz, A. Padilla, P. M. Saffin, Strong coupling in Horava gravity, JHEP 0908, 070 (2009) arXiv:0905.2579 [hep-th]].

[16] D. Garfinkle, T. Jacobson, A positive energy theorem for Einstein-aether and Hořava gravity, arXiv:1108.1835 [gr-qc]].

[17] A. E. Gumrukcuoglu, S. Mukohyama, Horava-Lifshitz gravity with $\lambda \rightarrow \infty$, Phys. Rev. D83, 124033 (2011). arXiv:1104.2087 [hep-th]].

[18] A. E. Gumrukcuoglu, S. Mukohyama, A. Wang, General relativity limit of Horava-Lifshitz gravity with a scalar field in gradient expansion, arXiv:1109.2609 [hep-th]].

[19] C. Herdeiro, S. Hirano and Y. Sato, n-DBI gravity, arXiv:1110.0832 [gr-qc].

[20] T. Jacobson, Extended Horava gravity and Einstein-aether theory, Phys. Rev. D81, 101502 (2010). arXiv:1001.4823 [hepthl].

[21] K. Koyama, F. Arroja, Pathological behaviour of the scalar graviton in Horava-Lifshitz gravity, JHEP 1003, 061 (2010) arXiv:0910.1998 [hep-th]].

[22] M. Li, Y. Pang, A Trouble with Horava-Lifshitz Gravity, JHEP 0908, 015 (2009) arXiv:0905.2751 [hep-th]].

[23] K. Lin, A. Wang, Q. Wu and T. Zhu, On strong coupling in nonrelativistic general covariant theory of gravity, Phys. Rev. D 84, 044051 (2011) arXiv:1106.1486 [hep-th]].

[24] Y. S. Myung, Massive graviton propagation of the deformed Hořava-Lifshitz gravity without projectability condition, Phys. Lett. B690, 526-533 (2010). arXiv:1004.2552 [hep-th]].

[25] H. Nastase, On IR solutions in Horava gravity theories, arXiv:0904.3604 [hep-th]].

[26] Y. C. Ong, P. Chen, Stability of Horava-Lifshitz Black Holes in the Context of AdS/CFT, arXiv:1106.3555 [hep-th]].

[27] A. Padilla, The good, the bad and the ugly... of Horava gravity, J. Phys. Conf. Ser. 259, 012033 (2010). arXiv:1009.4074 [hep-th]].

[28] A. Papazoglou, T. P. Sotiriou, Strong coupling in extended Horava-Lifshitz gravity, Phys. Lett. B685, 197-200 (2010). arXiv:0911.1299 [hep-th]].

[29] M. Pospelov, Y. Shang, On Lorentz violation in Horava-Lifshitz type theories, arXiv:1010.5249 [hep-th]].

[30] A. M. da Silva, An Alternative Approach for General Covariant Horava-Lifshitz Gravity and Matter Coupling, Class. Quant. Grav. 28, 055011 (2011). arXiv:1009.4885 [hep-th]].

[31] T. P. Sotiriou, M. Visser and S. Weinfurtner, Quantum gravity without Lorentz invariance, JHEP 0910, 033 (2009) arXiv:0905.2798 [hep-th]].

[32] T. P. Sotiriou, M. Visser, S. Weinfurtner, Phenomenologically viable Lorentz-violating quantum gravity, Phys. Rev. Lett. 102, 251601 (2009). arXiv:0904.4464 [hep-th]].

[33] T. P. Sotiriou, Horava-Lifshitz gravity: a status report, J. Phys. Conf. Ser. 283, 012034 (2011). arXiv:1010.3218 [hep-th]].

[34] T. Takahashi, J. Soda, Chiral Primordial Gravitational Waves from a Lifshitz Point, Phys. Rev. Lett. 102, 231301 (2009). arXiv:0904.0554 [hep-th]].

[35] M. Visser, Lorentz symmetry breaking as a quantum field theory regulator, Phys. Rev. D80, 025011 (2009). arXiv:0902.0590 [hep-th]].

[36] M. Visser, Status of Horava gravity: A personal perspective, J. Phys. Conf. Ser. 314, 012002 (2011). arXiv:1103.5587 [hep-th]].

[37] S. Weinfurtner, T. P. Sotiriou, M. Visser, Projectable Horava-Lifshitz gravity in a nutshell, J. Phys. Conf. Ser. 222, 012054 (2010) arXiv:1002.0308 [gr-qc]].

[38] A. Wang and Q. Wu, Stability of spin-0 graviton and strong coupling in Horava-Lifshitz theory of gravity, Phys. Rev. D 83, 044025 (2011) arXiv:1009.0268 [hep-th]].

[39] T. Zhu, F.-W. Shu, Q. Wu and A. Wang, General covariant Horava-Lifshitz gravity without projectability condition and its applications to cosmology, arXiv:1110.5106 [hep-th].

[40] R. Brandenberger, Matter Bounce in Horava-Lifshitz Cosmology, Phys. Rev. D80, 043516 (2009). arXiv:0904.2835 [hepth]].

[41] S. Dutta and E. N. Saridakis, Observational constraints on Horava-Lifshitz cosmology, JCAP 1001, 013 (2010) arXiv:0911.1435 [hep-th]].

[42] C. Furtado, J. R. Nascimento, A. Y. .Petrov, A. F. Santos, Horava-Lifshitz gravity and Godel Universe, Phys. Rev. D84, 047702 (2011). arXiv:1106.4003 [hep-th]].

[43] X. Gao, Y. Wang, R. Brandenberger, A. Riotto, Cosmological Perturbations in Horava-Lifshitz Gravity, Phys. Rev. D81, 083508 (2010). arXiv:0905.3821 [hep-th]].

[44] S. Mukohyama, Horava-Lifshitz Cosmology: A Review, Class. Quant. Grav. 27 (2010). arXiv:1007.5199 [hep-th]].

[45] E. N. Saridakis, Aspects of Horava-Lifshitz cosmology, Int. J. Mod. Phys. D 20, 1485 (2011) arXiv:1101.0300 [astro-ph.CO]].

[46] A. Wang and Y. Wu, Thermodynamics and classification of cosmological models in the Horava-Lifshitz theory of gravity, 
JCAP 0907, 012 (2009) arXiv:0905.4117 [hep-th]].

[47] A. Wang and R. Maartens, Linear perturbations of cosmological models in the Horava-Lifshitz theory of gravity without detailed balance, Phys. Rev. D 81, 024009 (2010) arXiv:0907.1748 [hep-th]].

[48] R. J. Rivers, Nuovo Cimento 34 (1964) 387.

[49] D. E. Neville, A Gravity Lagrangian With Ghost Free Curvature**2 Terms, Phys. Rev. D 18, 3535 (1978).

[50] E. Sezgin and P. van Nieuwenhuizen, New Ghost Free Gravity Lagrangians with Propagating Torsion, Phys. Rev. D 21, 3269 (1980).

[51] C. A. Hernaski, A. A. Vargas-Paredes and J. A. Helayel-Neto, A Discussion on Massive Gravitons and Propagating Torsion in Arbitrary Dimensions, Phys. Rev. D 80, 124012 (2009) arXiv:0905.1068 [hep-th]].

[52] C. A. Hernaski, B. Pereira-Dias and A. A. Vargas-Paredes, Extending the Spin Projection Operators for Gravity Models with Parity-Breaking in 3-D, Phys. Lett. A 374, 3410 (2010) arXiv:1001.2784 [hep-th]].

[53] J. A. Helayel-Neto, C. A. Hernaski, B. Pereira-Dias, A. A. Vargas-Paredes and V. J. Vasquez-Otoya, Chern-Simons Gravity with (Curvature) $)^{2}$ - and (Torsion) ${ }^{2}$-Terms and A Basis of Degree-of-Freedom Projection Operators, Phys. Rev. D 82, 064014 (2010), arXiv:1005.3831 [hep-th].

[54] B. Pereira-Dias, C. A. Hernaski, and J. A. Helayel-Neto, Probing the Effects of Lorentz-Symmetry Violating Chern-Simons and Ricci-Cotton Terms in Higher Derivative Gravity, Phys. Rev. D 83, 084011 (2011) [arXiv:1009.5132 [hep-th]].

[55] K.S. Stelle, Renormalization of Higher Derivative Quantum Gravity, Phys. Rev. D 16, 953 (1977).

[56] Y. Iwasaki, Consistency condition for propagators, Phys. Rev. D2, 2255-2256 (1970).

[57] H. van Dam, M. J. G. Veltman, Massive and massless Yang-Mills and gravitational fields, Nucl. Phys. B22, 397-411 (1970).

[58] V. I. Zakharov, Linearized gravitation theory and the graviton mass, JETP Lett. 12, 312 (1970).

[59] A. I. Vainshtein, To the problem of nonvanishing gravitation mass, Phys. Lett. B39, 393-394 (1972).

[60] P. Van Nieuwenhuizen, Radiation of massive gravitation, Phys. Rev. D7, 2300-2308 (1973)

[61] K. Hinterbichler, Theoretical Aspects of Massive Gravity, arXiv:1105.3735 [hep-th]].

[62] J. L. Anderson, Principles of Relativity Physics (Academic, New York, 1967).

[63] P. de Aquino, K. Hagiwara, Q. Li and F. Maltoni, Simulating graviton production at hadron colliders, JHEP 1106, 132 (2011) arXiv:1101.5499 [hep-ph]].

[64] S. Ask, Simulation of $Z$ plus Graviton/Unparticle Production at the LHC, Eur. Phys. J. C 60, 509 (2009) arXiv:0809.4750 [hep-ph]].

[65] X. Gao, C. S. Li, J. Gao, J. Wang and R. J. Oakes, Next-to-leading order QCD predictions for graviton and photon associated production in the Large Extra Dimensions model at the LHC, Phys. Rev. D 81, 036008 (2010) arXiv:0912.0199 [hep-ph]].

[66] A. S. Goldhaber and M. M. Nieto, Photon and Graviton Mass Limits, Rev. Mod. Phys. 82, 939 (2010) arXiv:0809.1003 [hep-ph]].

[67] K. Hagiwara, P. Konar, Q. Li, K. Mawatari and D. Zeppenfeld, Graviton production with 2 jets at the LHC in large extra dimensions, JHEP 0804, 019 (2008) arXiv:0801.1794 [hep-ph]].

[68] S. Karg, M. Kramer, Q. Li and D. Zeppenfeld, NLO QCD corrections to graviton production at hadron colliders, Phys. Rev. D 81, 094036 (2010) arXiv:0911.5095 [hep-ph]].

[69] Z. Ya-Jin, M. Wen-Gan, H. Liang and Z. Ren-You, Associated production of graviton with e+ e- pair via photon-photon collisions at a linear collider, Phys. Rev. D 76, 054003 (2007) [arXiv:0708.1195 [hep-ph]]. 